\title{
Implantation of the Micra transcatheter pacing system: Single Polish center experience with the real costs of hospitalization analysis
}

\author{
Marcin Grabowski ${ }^{1}$, Marcin, Michalak ${ }^{1}$, Monika Gawałko ${ }^{1}$, Sylwia Gajda ${ }^{1}$, \\ Andrzej Cacko ${ }^{1}$, Łukasz Januszkiewicz ${ }^{1}$, Agnieszka Kołodzińska ${ }^{1}$, \\ Przemysław P. Mitkowski ${ }^{2}$, Gabor Z. Duray ${ }^{3}$, Grzegorz Opolski ${ }^{1}$ \\ ${ }^{1} 1^{\text {st }}$ Department of Cardiology, Medical University of Warsaw, Poland \\ ${ }^{2}$ Department of Cardiology, Poznan University of Medical Sciences, Poznan, Poland \\ ${ }^{3}$ Clinical Electrophysiology Department of Cardiology, Medical Center, \\ Hungarian Defence Forces, Budapest, Hungary
}

\begin{abstract}
Background: The Micra transcatheter pacing system (TPS) is a miniaturized, single-chamber pacemaker system. Study reported herein is an initial experience with implantation of the Micra TPS.

Methods: The leadless pacemaker was implanted in 10 patients with standard indications for a permanent pacemaker implantation. All hospitalization costs were calculated for all patients.

Results: The mean age of the patients was $75 \pm 7.1$ years, 6 were men and 4 were women. Four patients had permanent atrial fibrillation as the basal rhythm and 6 patients had sinus rhythm. All patients had at least one relative contraindication that precluded the use of a traditional pacing system. Mean intraoperative ventricular sensing amplitude was $10.6 \pm 5.4 \mathrm{mV}$, impedance $843 \pm 185 \mathrm{ohms}$, and pacing threshold at $0.24 \mathrm{~ms}$ was $0.56 \pm 0.23 \mathrm{~V}$. At discharge, those values were $13.9 \pm 5.6 \mathrm{mV}$, $667 \pm 119 \mathrm{ohms}$ and $0.47 \pm 0.17$, respectively. The mean duration of implantation procedure was $82 \mathrm{~min}$, while mean fluoroscopy time was $3.5 \mathrm{~min}$. Two patients developed hematoma at the groin puncture site post-implantation. In 1 case there was a need for erythrocyte mass transfusion and surgical intervention. Mean total time of hospitalization was 26 days and time from procedure to discharge 12 days. Average cost of hospitalization per 1 patient was 11,260.15 EUR minimal cost was 9,052.68 EUR, while maximal cost was 16,533.18 EUR.

Conclusions: Implantation of leadless pacemakers is feasible, safe and provides advantages over the conventional system. Hospitalization costs vary for individual patients in wide range. (Cardiol J 2020; 27, 1: 47-53)
\end{abstract}

Key words: leadless pacemakers, complications, procedure cost, hospitalization cost

\section{Introduction}

Recent advances in miniaturization technologies and battery chemistries have made it possible to develop a pacemaker small enough to implant within the heart while still aiming to provide similar effectiveness and durability to conventional pacemakers. The Micra transcatheter pacing system (Micra TPS) (Medtronic, USA) is a miniaturized single-chamber pacemaker system that is implanted directly to the right ventricle, eliminating the need for device pocket creation or insertion of a pacing lead, thereby avoiding some of the complications associated with traditional

Address for correspondence: Marcin Grabowski, MD, $\mathrm{PhD}, 1^{\text {st }}$ Department of Cardiology, Medical University of Warsaw, ul. Banacha 1a, 02-097 Warszawa, Poland, tel: +48 660751 816, fax: +48 2259919 57, e-mail: marcin.grabowski@wum.edu.pl

Received: 22.04.2018 Accepted: 12.08 .2018 
pacing systems $[1,2]$. This emerging technology has the potential to significantly improve outcomes associated with a need for long-term pacing and can help patients get back to work and limit disability or restrictions to lifestyle [3, 4].

In the present single-center observational study, an initial experience with implantation of the Micra TPS is reported.

\section{Methods}

\section{Procedure}

The Micra TPS is a single chamber ventricular pacemaker. The device is attached to a steerable catheter delivery system with catheter and is inserted through a femoral vein with the use of a 23-French (outer diameter $27 \mathrm{~F}$ ) introducer sheath. The delivery system is advanced into the right ventricle (RV), and the device is affixed to the myocardium with four electrically inactive nitinol tines located at the distal end of the device. If optimal electrical measurement results are not achieved the system is fully repositionable while the device is still connected to the delivery system. After verification of adequate electrical parameters and device fixation to the endocardium the device is released and delivery system is removed. According to this local strategy vascular access site was closed with subcutaneous absorbable double 'figure-of-eight' suture followed by $4 \mathrm{~h}$ bandage compression used for the access site in the groin [5].

Duration of procedure (from femoral vein puncture to venous access closure), fluoroscopy time, number of device repositions, periprocedural electrical measurements (sensing, threshold and impedance) and in-hospital adverse events related to procedure were evaluated.

\section{Patients}

All patients had classic indications for permanent pacing system implantation. Patients with sinus rhythm were not excluded if they had relative or absolute contraindication to traditional pacemaker implantation. Prior to procedure patients and their family members were informed of the characteristics of the new system, indications and potential complications. Informed consent was obtained.

\section{Costs of hospitalization analysis}

All costs of hospitalization were calculated and summed up for each patient. Costs were divided into following categories: Micra TPS device, medical materials excluding Micra TPS (disposable materials related to the procedure, pacemaker introducer), pharmaceuticals (e.g. oral drugs, antibiotics, disinfectants, analgesics), operating theatre staff (e.g. electrophysiologists, scrub nurse, personal costs of analgesia), cardiology department staff (e.g. cardiologists, nurses), additional laboratory tests (e.g. blood group, morphology, electrolytes, C-reactive protein, procalcitonin, natriuretic peptides, viral antigens and antibodies, clotting), additional non-laboratory tests (e.g. echocardiography, X-ray), additional non-medical costs (e.g. materials and energy, linen, maintenance materials, office supplies, informatics and information technology, laboratory reagents, medical gases, electricity, heat, water, permanent foreign services, minor repair of hardware, postage and telephone charges - non-medical indirect costs, management).

\section{Results}

\section{Baseline characteristics}

The Micra TPS implantation was attempted in 10 patients with $100 \%$ success rate. All patients had standard indication for a permanent pacemaker implantation, i.e. third-degree atrioventricular block (40\%), second-degree atrioventricular block (30\%), symptomatic sick sinus syndrome (20\%), bradycardia-tachycardia syndrome $(10 \%)$. The mean age of patients was $75 \pm 7.6$ years, 6 were men and 4 were women. Four patients had permanent atrial fibrillation (AF) as basal rhythm and 4 patients had paroxysmal AF or atrial flutter. Over half of the patients had a previously implanted cardiac electronic device including cardiac resynchronization therapy. In addition, all patients had at least one condition that precluded the use of a traditional pacing system, i.e. history of implantable cardiac electronic device (ICED) related infection (60\%), lack of vascular access on one site and the need to preserve venous system for hemodialysis on opposite site (20\%) and post mastectomy bilateral upper limb lymphedema (10\%). Patien characteristics and basic procedural data are summarized in Table 1.

\section{Procedure}

All the devices were implanted through the right femoral vein to the septum of RV. In $50 \%$ of patient there was no need for any repositioning of the system and the position of the device had to be changed $\geq 2$ times only in 2 patients due to suboptimal pacing threshold or sensing value. Mean procedure time in the present population was $82 \mathrm{~min}$ (from femoral vein puncture to vascular 


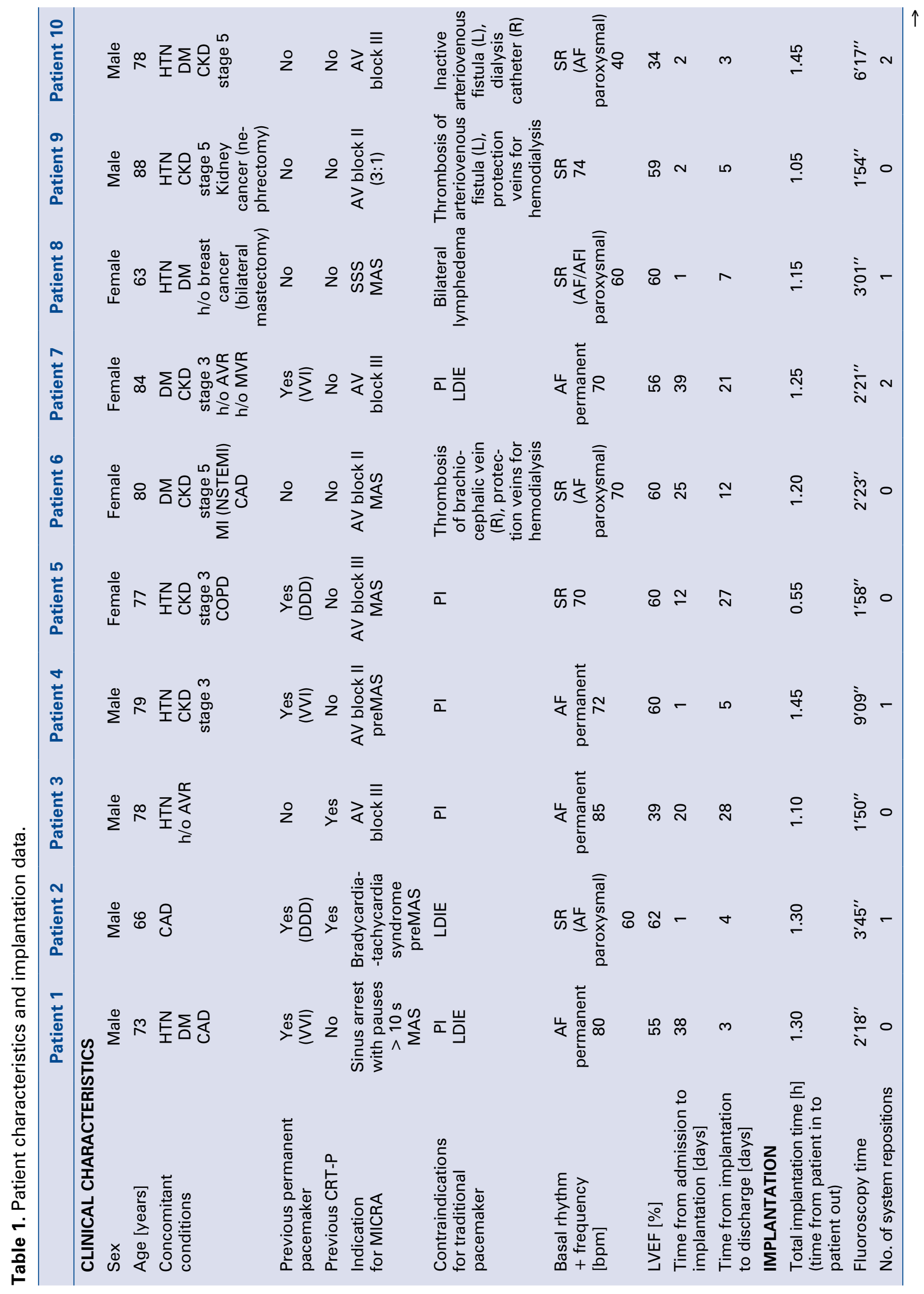




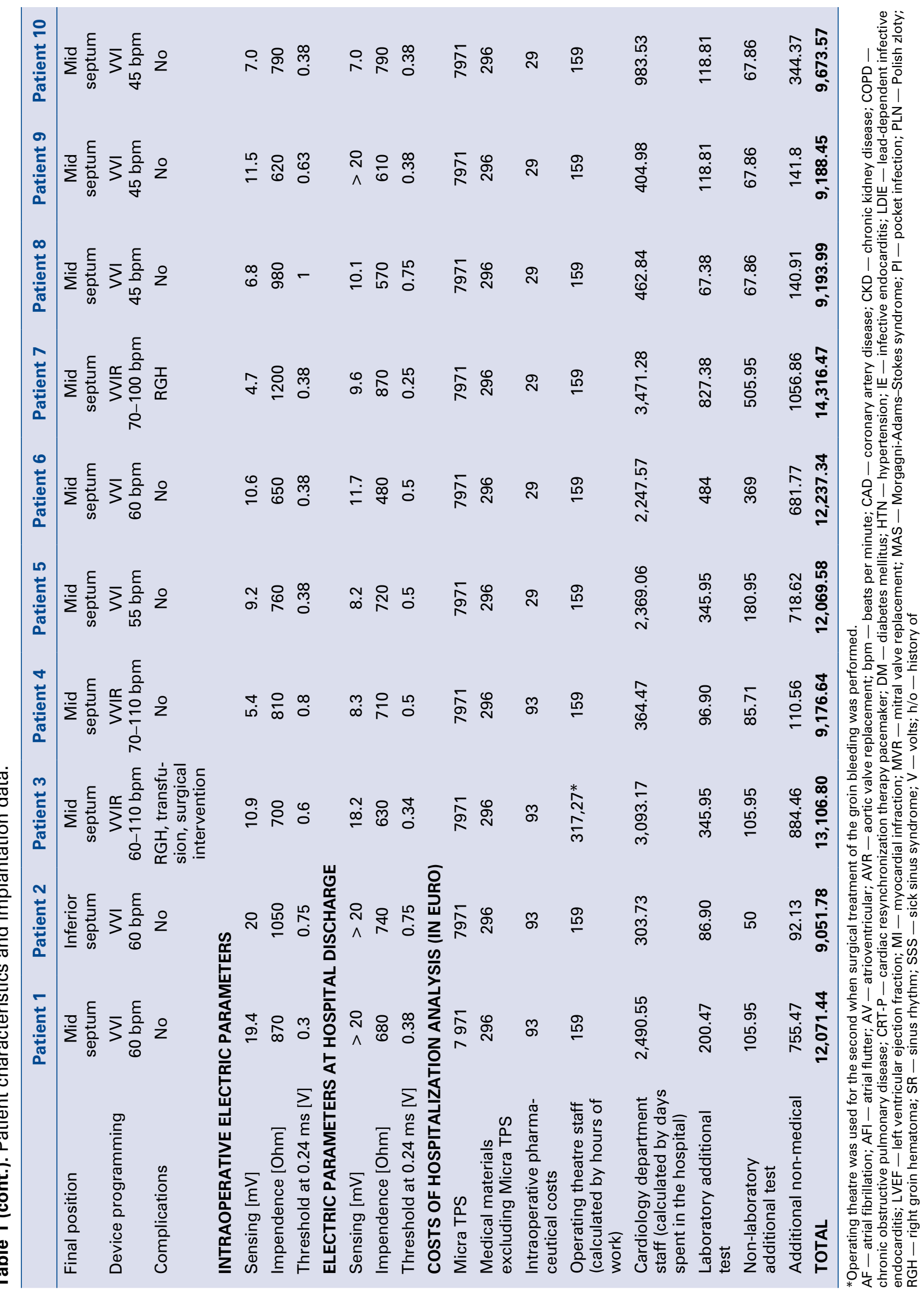


sheath removal) and mean fluoroscopy duration was $3.5 \mathrm{~min}$. Mean procedure (from introducer insertion to introducer removal) and fluoroscopy time in post-approval registry was $34.8 \mathrm{~min}$ and 8.9 min, respectively.

The mean intraoperative sensing value was $10.6 \pm 5.4 \mathrm{mV}$ and the impedance was $843 \pm 185$ ohms. At discharge from hospital, those values were $13.9 \pm 5.6 \mathrm{mV}$ and $667 \pm 119 \mathrm{ohms}$, respectively. The recommended pacing threshold value, i.e. $\leq 1 \mathrm{~V}$ at $0.24 \mathrm{~ms}$ was achieved in all patients. Mean procedure duration was $82 \mathrm{~min}(55-90 \mathrm{~min})$, while mean fluoroscopy time was $3.5 \mathrm{~min}$ (minimal 1'50" - maximal 9'09').

Mean total time of hospitalization was 26 days (5-60 days) and time from procedure to discharge 12 days (3-21 days). During post-implantation period $2(20 \%)$ patients developed hematoma at the groin puncture site. In 1 case there was a need for erythrocyte mass transfusion and surgical intervention. The second one was treated conservatively without any sequelae.

\section{Adverse events}

Two patients developed groin hematoma. The first patient developed large hematoma that was associated with anemization, required blood transfusion (6 units of blood) and surgical intervention. The second patient complained of groin pain.

Ultrasound imaging revealed relatively small hematoma that was absorbed spontaneously. What should be underlined, both patients had a history of valve replacement (mechanical aortic prosthesis in $1^{\text {st }}$ case, mechanical aortic and mitral valve in $2^{\text {nd }}$ case) and were under bridging anticoagulant therapy (low molecular weight heparin). In patients receiving vitamin $\mathrm{K}$ antagonists (VKA), treatment was continued until the international normalization rate was therapeutic (range of 2-3) and in patients on non-VKA, treatment discontinued at least $24 \mathrm{~h}$ before operation.

\section{Costs of hospitalization analysis}

Real costs of hospitalization for every patient are presented in Table 1. Average cost of hospitalization per 1 patient was 11,260.15 EUR (minimal $=9051.68$ EUR and maximal = 16,533.18 EUR). Average costs for each category were as follows: 8,267.66 EUR for medical materials; 54.58 EUR for pharmaceuticals; 174.5 EUR for operating theatre staff; 1,619.12 EUR (minimal = 303.73 EUR and maximal $=3,471.28$ EUR) for cardiology department staff; 269.26 EUR (minimal $=67.38$ EUR and maximal $=827.38$ EUR) for laboratory additional test, 160.71 EUR for non-laboratory additional test; 492.69 EUR for additional non-medical costs.

\section{Discussion}

Elimination of leads and pocket with the introduction of leadless pacemakers offered potential advantages over conventional transvenous systems. Lead- and pocket-related complications are dominant adverse events associated with cardiac pacing $[1,2]$. Pacing leads and the pacemaker as a high-volume foreign body become the background for CIED related infections that are associated with poor prognosis despite complete hardware removal [6, 7]. Micra's small size, reduced surface area, and lack of lead exposed to the bloodstream appear to substantially mitigate the risk of early device infection [8]. Over the long-term follow-up, these features will also promote complete device encapsulation, which may significantly reduce the risk of late infections.

Micra TPS is a full capability VVIR pacemaker. Typical indications for this system include patients with atrioventricular conduction disturbances and permanent AF. Despite that fact more and more patients are offered with the leadless system because of conditions that precludes implantation of conventional pacemaker such as history or high risk of infection, lack of axillary/subclavian vascular access, thrombosis or need to preserve the venous system for hemodialysis. This group of patients amounted $6.2 \%$ in Investigational Device Exemption (IDE) study and reached $20.9 \%$ in postapproval registry $[9,10]$. In our cohort all patients, had at least one factor that precluded implantation of transvenous pacing system.

An early report of Micra TPS implantations showed very high procedural success rate of $100 \%$ [3]. It was reduced to $99.2 \%$ in a full cohort of patients in the IDE study [9]. The interim report from Micra TPS post-approval registry also showed high procedural efficacy with $99.6 \%$ successful implantations [10]. All 10 implantation attempts were completed in this study. All the devices were able to be implanted to the RV septum, which was confirmed in all patients in LAO projection with contrast medium injection. Septal positioning of the system seems to bring some benefits in terms of avoiding pericardial effusion and tamponade. In the literature a trend toward more frequent septal implantations could be observed. There were $65.9 \%$ apical implantations in the IDE study compared to $39.3 \%$ in post-approval registry $[8,9]$. A similar trend could be observed with a different 
transcatheter pacemaker, Nanostim (apical position in primary analysis cohort vs. total cohort, $48.4 \%$ vs. $38.1 \%$, respectively) [11].

Although the purpose of each case was a RV septal pacing, it did not translate to significantly longer procedure duration and/or fluoroscopy time. Recommended electrical parameters in 9 patients were achieved. In 1 patient (patient no. 7) after two repositions of the system the procedure was ended with sensing value slightly lower than recommended, i.e. $4.7 \mathrm{mV}$. In accordance to observations from the trials and registry the value increased and reached $9.6 \mathrm{mV}$ before hospital discharge $[9,10,12]$.

Nevertheless leadless pacing reduces the rate of some procedural and long-term complications it also brings new problems that were not present with traditional pacing systems, i.e. vascular complications at the groin puncture site. In the IDE study arteriovenous fistula or pseudoaneurysm occurred in $5(0.7 \%)$ patients [8]. A similar rate of vascular complications was observed in postapproval registry. Among total $0.75 \%$ of access site complications, there were 2 hematomas $(0.25 \%$ of patients) [9]. Currently there is no data about proper periprocedural antithrombotic management in those patients.

Although implantation of single chamber VVI pacemaker is on the list of guaranteed services but total cost of Micra TPS highly exceeds reimbursement level for this category, so individual financing was implemented for each patient and this study depicts expenditures divided into a range of categories. According to available research this is the first cost analysis of the Micra implantation procedure. Hospitalization costs for individual patient with a wide range of medical conditions. The price of the Micra device was the same for all procedures, but final costs varied depending on patient. Those who had an infection or an implanted device extraction had higher expenditures than those with simple/ stand alone Micra implantation procedure. Two patients had bleeding complications. One patient had pseudoaneurysm in the vascular access site, while the other had femoral artery aneurysm demanding intervention. These events prolonged hospitalization length and therefore final costs. The relatively low cost of operating theatre staff also deserves comment. This is due to the fact that the hospital calculates it from the staff costs based on the hourly wage rates, that, while calculating the actual time of treatment, gives very small amounts and does not take into account the time between procedures. In addition, current analysis did not include costs of proctors presence during first 6 procedures. According to the hospital contract these costs were covered by the device supplier.

\section{Limitations of the study}

The cost of Micra implantation varies dramatically between centers, contracts with the vendor, and country which limits the generalizability of this report. However, the primary objective of this study was to compare hospital costs between patients with different clinical profiles assuming one price of the device, mainly due to the fact that Poland is applying for Micra implantation reimbursement. Therefore a comparison was not made with a matched group undergoing transvenous pacemaker implantation, because the aim herein was not a comparison in the context of effectiveness and safety assessment between tranvenous and leadless pacemaker.

The first ten cases of Micra implantation were performed in the certificated Clinic. The introduction of a novel technology is usually accompanied by a period of learning in which operators develop and refine new skills until they achieve a "steady state" characterized by high efficiency and procedural success with low complications. This is one limitation of the present study.

\section{Conclusions}

The presented registry of Micra implantation is the first single-center observational study in Poland. Early results from this and other clinical evaluations suggest that leadless pacing is effective, safe and could gain wider adoption particularly in patients with contraindications to conventional cardiac pacing. Further studies on periprocedural antithrombotic management in patients with indications to permanent anticoagulation are warranted. Hospitalization costs for individual patients varies over a large range. Diversity of costs is mainly attributed to concomitant indications (e.g. infection), comorbidities (e.g. dialysis) and post-procedural complications (e.g. bleeding and hematoma).

Conflict of interest: Marcin Grabowski has honoraria from Medtronic.

\section{References}

1. Udo EO, Zuithoff NPA, van Hemel NM, et al. Incidence and predictors of short- and long-term complications in pacemaker therapy: the FOLLOWPACE study. Heart Rhythm. 2012; 9(5): 728-735, doi: 10.1016/j.hrthm.2011.12.014, indexed in Pubmed: 22182495 . 
2. Kirkfeldt RE, Johansen JB, Nohr EA, et al. Complications after cardiac implantable electronic device implantations: an analysis of a complete, nationwide cohort in Denmark. Eur Heart J. 2014; 35(18): 1186-1194, doi: 10.1093/eurheartj/eht511, indexed in Pubmed: 24347317.

3. Ritter P, Duray GZ, Steinwender C, et al. Early performance of a miniaturized leadless cardiac pacemaker: the Micra Transcatheter Pacing Study. Eur Heart J. 2015; 36(37): 2510-2519, doi: 10.1093/eurheartj/ehv214, indexed in Pubmed: 26045305.

4. Knops RE, Tjong FVY, Neuzil P, et al. Chronic performance of a leadless cardiac pacemaker: 1-year follow-up of the LEADLESS trial. J Am Coll Cardiol. 2015; 65(15): 1497-1504, doi: 10.1016/j. jacc.2015.02.022, indexed in Pubmed: 25881930.

5. El-Chami MF, Roberts PR, Kypta A, et al. How to Implant a Leadless Pacemaker With a Tine-Based Fixation. J Cardiovasc Electrophysiol. 2016; 27(12): 1495-1501, doi: 10.1111/jce.13092, indexed in Pubmed: 27600684.

6. Sohail MR, Hussain S, Le KY, et al. Management and outcome of permanent pacemaker and implantable cardioverter-defibrillator infections. J Am Coll Cardiol. 2007; 49(18): 1851-1859, doi: 10.1016/j.jacc.2007.01.072, indexed in Pubmed: 17481444.

7. Baddour LM, Epstein AE, Erickson CC, et al. Update on cardiovascular implantable electronic device infections and their man- agement: a scientific statement from the American Heart Association. Circulation. 2010; 121(3): 458-477, doi: 10.1161/CIRCULATIONAHA.109.192665, indexed in Pubmed: 20048212.

8. Kypta A, Blessberger H, Kammler J, et al. Leadless cardiac pacemaker implantation after lead extraction in patients with severe device infection. J Cardiovasc Electrophysiol. 2016; 27(9): 1067-1071, doi: 10.1111/jce.13030, indexed in Pubmed: 27296508.

9. Reynolds D, Duray G, Omar R, et al. A Leadless Intracardiac Transcatheter Pacing System. N Engl J Med. 2016; 374(6): 533-541, doi: 10.1056/nejmoa1511643.

10. Roberts PR, Clementy N, Al Samadi F, et al. A leadless pacemaker in the real-world setting: The Micra Transcatheter Pacing System Post-Approval Registry. Heart Rhythm. 2017; 14(9): 1375-1379, doi: 10.1016/j.hrthm.2017.05.017, indexed in Pubmed: 28502871.

11. Reddy VY, Exner DV, Cantillon DJ, et al. Percutaneous Implantation of an Entirely Intracardiac Leadless Pacemaker. N Engl J Med. 2015; 373(12): 1125-1135, doi: 10.1056/NEJMoa1507192, indexed in Pubmed: 26321198.

12. Duray GZ, Ritter P, El-Chami M, et al. Long-term performance of a transcatheter pacing system: 12-Month results from the Micra Transcatheter Pacing Study. Heart Rhythm. 2017; 14(5): 702-709, doi: 10.1016/j.hrthm.2017.01.035, indexed in Pubmed: 28192207. 